Published in final edited form as:

Conf Proc IEEE Eng Med Biol Soc. 2009 ; 2009: 1852-1855. doi:10.1109/IEMBS.2009.5332622.

\title{
Pilot Canine Investigation of the Cardiopulmonary Baroreflex Control of Ventricular Contractility
}

\author{
Javier A. Sala-Mercado, \\ Department of Physiology, Wayne State University School of Medicine, Detroit, MI 48201 USA \\ (javosala@hotmail.com).
}

Xiaoxiao Chen,

Department of Electrical and Computer Engineering, Michigan State University, East Lansing, MI 48824 USA (chenxia7@msu.edu).

\section{Robert L. Hammond,}

Department of Physiology, Wayne State University School of Medicine, Detroit, MI 48201 USA

(robert.hammond@beaumont.edu).

Jong-Kyung Kim,

Department of Physiology, Wayne State University School of Medicine, Detroit, MI 48201 USA

(jkkim@ucdavis.edu).

Phillip J. McDonald,

Department of Surgery, Wayne State University School of Medicine, Detroit, MI 48201 USA

Larry W. Stephenson,

Department of Surgery, Wayne State University School of Medicine, Detroit, MI 48201 USA

Donal S. O'Leary, and

The Department of Physiology, Wayne State University School of Medicine, Detroit, MI 48201 USA (doleary@med.wayne.edu).

\section{Ramakrishna Mukkamala [Member, IEEE]}

Department of Electrical and Computer Engineering, Michigan State University, East Lansing, MI 48824 USA (phone: 517-353-3120; fax: 517-353-1980; rama@egr.msu.edu).

\section{Abstract}

We performed a pilot investigation of the cardiopulmonary baroreflex control of ventricular contractility in two conscious dogs. We specifically measured spontaneous beat-to-beat hemodynamic variability before and after the administration of propranolol. We then identified the transfer function relating beat-to-beat fluctuations in central venous pressure (CVP) to maximal ventricular elastance $\left(\mathrm{E}_{\max }\right)$ to characterize the cardiopulmonary baroreflex control of ventricular contractility, while accounting for the influences of arterial blood pressure fluctuations on $\mathrm{E}_{\max }$ via the arterial baroreflex and heart rate fluctuations on $E_{\max }$ via the force-frequency relation. Our major finding is that the cardiopulmonary baroreflex responds to an increase (decrease) in CVP by increasing (decreasing) $\mathrm{E}_{\max }$ via the $\beta$-sympathetic nervous system.

\section{INTRODUCTION}

The baroreflex systems are primarily responsible for maintaining blood pressures over short time scales of seconds to minutes. It is well known that the arterial baroreflex senses arterial blood pressure (ABP) via baroreceptors lying in the carotid sinus and aortic arch and buffers a decrease (increase) in ABP by increasing (decreasing) heart rate (HR), total peripheral resistance, and ventricular contractility. However, the cardiopulmonary baroreflex is less 
understood. The sensory receptors of this system are more complex, residing mainly in the cardiac chambers but also in the walls of the pulmonary artery [1]. These receptors have been shown to be very responsive to central venous pressure (CVP) [2,3]. The cardiopulmonary baroreflex responds to a change in CVP by inducing an opposite change in total peripheral resistance [3,4]. An increase in CVP also leads to an increase in HR (i.e., Bainbridge effect) in dogs [5], but an opposite change may occur in humans [2].

To our knowledge, the cardiopulmonary baroreflex control of ventricular contractility has not been elucidated. A change in CVP could induce a same directional change in ventricular contractility so as to maintain CVP, much like the Bainbridge effect. On the other hand, a change in CVP could cause an opposite change in ventricular contractility in order to blunt the forthcoming change in ABP due to the altered preload, much like the cardiopulmonary baroreflex control of total peripheral resistance.

We performed a pilot canine investigation of the cardiopulmonary baroreflex control of ventricular contractility. More specifically, we measured spontaneous beat-to-beat hemodynamic variability from two conscious dogs during control conditions and from one of the dogs after the administration of propranolol to abolish the neural control of ventricular contractility. We then identified the transfer function relating beat-to-beat fluctuations in CVP to maximal ventricular elastance $\left(\mathrm{E}_{\max }\right)$, which is perhaps the best available index of ventricular contractility [6-8], while accounting for other influences on $\mathrm{E}_{\max }$ including ABP fluctuations via the arterial baroreflex.

\section{METHODS}

\section{A. Data Collection}

We collected pilot hemodynamic data for this study from two adult dogs $(20-25 \mathrm{~kg})$ in the context of performing experiments to address unrelated specific aims. We describe only those aspects of the experimental procedures that were relevant to the present study. All procedures were reviewed and approved by the Wayne State University Animal Investigator Committee.

We studied each dog on three experimental days with suitable recovery periods in between as follows. On the first day, we performed a midline sternotomy under sterile conditions. We installed instrumentation including two pairs of sonomicrometry crystals (Sonometrics) on the left ventricular (LV) endocardium for continuous LV volume (LVV) as described in [9]; a fully implanted system with a micromanometer-tipped catheter (Data Sciences International) in the left ventricle (LV) for continuous LV pressure (LVP); an ultrasonic flow probe (Transonic Systems) around the ascending aorta for continuous cardiac output; and hydraulic vascular occluders (In Vivo Metrics) on the superior and inferior vena cava to diminish cardiac preload. Then, on the second day, we installed additional instrumentation under sterile conditions including fluid-filled catheters in the terminal aorta for continuous $\mathrm{ABP}$ and in the right atrium for continuous CVP. Finally, on the third day, we recorded the cardiovascular measurements during a baseline period of 7-14 min and transient vena cava occlusion while the dogs were standing quietly. For the second dog, we then administered propranolol to achieve complete $\beta$-sympathetic nervous blockade and likewise recorded the measurements.

\section{B. Data Analysis}

We analyzed the hemodynamic data for the three conditions of the two dogs. First, we determined $\mathrm{E}_{\max }$ and $\mathrm{CVP}$, along with $\mathrm{ABP}$ and $\mathrm{HR}$, on a beat-to-beat basis from the continuous measurements during the baseline period. Then, we identified the transfer function relating the spontaneous beat-to-beat fluctuations in $\mathrm{CVP}$ to $\mathrm{E}_{\max }\left(\mathrm{CVP} \rightarrow \mathrm{E}_{\max }\right)$, while effectively eliminating the known influences of beat-to-beat $A B P$ and $H R$ fluctuations on $E_{\max }$. 
More specifically, as described in a companion study of dynamic $\mathrm{E}_{\max }$ control before and after heart failure [10], we estimated beat-to-beat $\mathrm{E}_{\max }$ during the baseline period according to the procedure shown in Fig. 1. First, we applied the traditional method for determining $\mathrm{E}_{\max }$ by performing linear regression on the end-systolic LVP-LVV points during the transient vena cava occlusion [8]. The slope of the resulting line represents the average $E_{\max }$, while the $x-$ intercept indicates the $\mathrm{LV}$ unstressed volume $\left(\mathrm{V}_{0}\right)$. Then, assuming constant $\mathrm{V}_{0}$, we computed the time-varying LV elastance (LVE) curve from the continuous LVP and LVV during the baseline period by dividing the former measurement by the difference between the latter measurement and $\mathrm{V}_{0}$. Finally, we determined $\mathrm{E}_{\max }$ on a beat-to-beat basis by identifying the maximum of the LVE curve over each beat.

We computed beat-to-beat CVP and ABP by respectively time averaging the continuous CVP and $\mathrm{ABP}$ over each beat during the baseline period and identified beat-to-beat $\mathrm{HR}$ from the continuous cardiac output during the same period. We then re-sampled the $\mathrm{E}_{\max }, \mathrm{CVP}, \mathrm{ABP}$, and $\mathrm{HR}$ beat series to time series at a sampling frequency of $1 \mathrm{~Hz}$ as described in [11].

We identified the $\mathrm{CVP} \rightarrow \mathrm{E}_{\max }$ transfer function by analyzing all four of the time series according to the block diagram illustrated in Fig. 2. As indicated in this diagram, we simultaneously identified the transfer function relating beat-to-beat fluctuations in ABP to $\mathrm{E}_{\max }\left(\mathrm{ABP} \rightarrow \mathrm{E}_{\max }\right)$, which characterizes the arterial baroreflex, and the transfer function relating beat-to-beat fluctuations in $H R$ to $\mathrm{E}_{\max }\left(\mathrm{HR} \rightarrow \mathrm{E}_{\max }\right)$, which characterizes the forcefrequency relation. In this way, the influence of beat-to-beat fluctuations in CVP on $\mathrm{E}_{\max }$ was isolated from other major confounding factors. We also estimated the perturbing noise source $\mathrm{N}_{\mathrm{E}_{\max }}$ in the block diagram, which represents the residual variability in $\mathrm{E}_{\max }$ not explained by the analyzed fluctuations. In particular, we mathematically represented the block diagram with the following autoregressive exogenous input structure:

$\Delta \mathrm{E}_{\max }(\mathrm{t})=\sum_{\mathrm{i}=1}^{\mathrm{p}} \mathrm{a}_{\mathrm{i}} \cdot \Delta \mathrm{E}_{\max }(\mathrm{t}-\mathrm{i})+\sum_{\mathrm{i}=\mathrm{q}}^{\mathrm{r}} \mathrm{b}_{\mathrm{i}} \cdot \Delta \mathrm{CVP}(\mathrm{t}-\mathrm{i})+\sum_{\mathrm{i}=\mathrm{s}}^{\mathrm{m}} \mathrm{c}_{\mathrm{i}} \cdot \Delta \mathrm{ABP}(\mathrm{t}-\mathrm{i})+\sum_{\mathrm{i}=0}^{\mathrm{n}} \mathrm{d}_{\mathrm{i}} \cdot \Delta \mathrm{HR}(\mathrm{t}-\mathrm{i})+\mathrm{W}_{\mathrm{E}_{\max }}(\mathrm{t})$

where $t$ is discrete time; the four sets of unknown parameters $\left\{a_{i}, b_{i}, c_{i}, d_{i}\right\}$ fully define the three transfer functions; the unmeasured residual error $\mathrm{W}_{\mathrm{E}_{\max }}$ together with the set of parameters $\left\{\mathrm{a}_{\mathrm{i}}\right\}$ specify $\mathrm{N}_{\mathrm{E}_{\max }}$; and the unknown model order, $\mathrm{p}, \mathrm{q}, \mathrm{r}, \mathrm{s}, \mathrm{m}$, and $\mathrm{n}$, limit the number of parameters [12]. For a fixed model order, we analytically estimated the parameters from zero-mean fluctuations in the $1 \mathrm{~Hz} C \mathrm{CP}, \mathrm{ABP}, \mathrm{HR}$, and $\mathrm{E}_{\max }$ time series by linear least squares minimization of $\mathrm{W}_{\mathrm{E}_{\max }}$ [12]. We set $\mathrm{p}, \mathrm{r}$, and $\mathrm{m}$ to two, $\mathrm{q}$, and $\mathrm{s}$, respectively, on the basis of a compelling previous study demonstrating that the arterial baroreflex control of $\mathrm{E}_{\max }$ could be well represented as a second-order delay system $[13,14]$. We determined q, s, and $\mathrm{n}$ by minimization of the popular minimum description length criterion [12].

\section{RESULTS}

Figs. 3 and 4 illustrate the $\mathrm{CVP} \rightarrow \mathrm{E}_{\max }, \mathrm{ABP} \rightarrow \mathrm{E}_{\max }$, and $\mathrm{HR} \rightarrow \mathrm{E}_{\max }$ transfer functions in terms of intuitive step responses, respectively, from the first dog during the control condition and the second dog during the control and propranolol conditions. During the control condition, both $\mathrm{CVP} \rightarrow \mathrm{E}_{\max }$ step responses indicate that $\mathrm{E}_{\max }$ would increase in response to a step increase in CVP. Quantitatively, the average gain value and dominant time constant of these steps responses are $0.2097 \mathrm{ml}^{-1}$ and $11.17 \mathrm{sec}$. The corresponding pairs of $\mathrm{ABP} \rightarrow \mathrm{E}_{\max }$ and $\mathrm{HR} \rightarrow \mathrm{E}_{\max }$ step responses show that $\mathrm{E}_{\max }$ would decrease and increase, respectively, in response to step increases in $A B P$ and $H R$. The average gain value of the $A B P \rightarrow E_{\max }$ step response and the average dominant time constant of the $\mathrm{HR} \rightarrow \mathrm{E}_{\max }$ step response are smaller than those of the $\mathrm{CVP} \rightarrow \mathrm{E}_{\max }$ step response. During the propranolol condition, the 
$\mathrm{CVP} \rightarrow \mathrm{E}_{\max }$ step response indicates that $\mathrm{E}_{\max }$ would not change in response to a step increase in CVP. The corresponding $\mathrm{ABP} \rightarrow \mathrm{E}_{\max }$ and $\mathrm{HR} \rightarrow \mathrm{E}_{\max }$ step responses similarly reveal blunted responses to steps increases in $\mathrm{ABP}$ and $\mathrm{HR}$.

\section{DISCUSSION}

In summary, we have investigated the cardiopulmonary baroreflex control of ventricular contractility in terms of $\mathrm{E}_{\max }$ in two conscious dogs. Our results suggest that, similar to the Bainbridge effect, the cardiopulmonary baroreflex responds to an increase (decrease) in CVP by increasing (decreasing) $\mathrm{E}_{\max }$. This mechanism appears to be mediated by the $\beta$-sympathetic nervous system, as the $\mathrm{E}_{\max }$ response to a change in CVP was abolished following the administration of propranolol. These pilot results may be amongst, if not, the first to illustrate how the cardiopulmonary baroreflex controls ventricular contractility.

Further, our ancillary results here on the $\mathrm{E}_{\max }$ response to changes in ABP and HR are consistent with known physiology. In particular, we found that an ABP change produces an opposing change in $\mathrm{E}_{\max }$, which is consistent with the negative feedback dynamics of the arterial baroreflex, while a HR change induces the same directional change in $\mathrm{E}_{\max }$, which is congruent with the force-frequency relation. Moreover, the $\mathrm{E}_{\max }$ response to a HR change was markedly faster than to an ABP change. This result is in accord with the force-frequency relation being mediated by fast mechanical effects and the arterial baroreflex being governed by the sluggish sympathetic nervous system. Finally, as expected, the $\mathrm{E}_{\max }$ response to a change in $\mathrm{ABP}$ was eliminated after the administration of propranolol. (However, it is unclear why the $\mathrm{E}_{\max }$ response to a $\mathrm{HR}$ change was also blunted after the drug administration.) These physiologically consistent results add confidence to our new findings on the cardiopulmonary baroreflex control of ventricular contractility.

Our future efforts will focus on confirming the results reported herein in a larger number of animals. Ultimately, we believe that this line of research will translate to a significant advance in the understanding of baroregulatory physiology.

\section{Acknowledgments}

This work was supported by the National Institutes of Health (HL-55473, HL-080568) and the National Science Foundation (CAREER 0643477).

\section{REFERENCES}

1. Bishop, VS.; Malliani, A.; Thoren, P. Handbook of Physiology. The Cardiovascular System. Peripheral Cierculation and Organ Blood Flow. Bethesda, MD: Am. Physiol. Soc.; 1983. Cardiac mechanoreceptors; p. 497-555.

2. Desai TH, Collins JC, Snell M, et al. Modeling of arterial and cardiopulmonary baroreflex control of heart rate. Am. J. Physiol 1997; vol. 272:H2343-H2352. [PubMed: 9176304]

3. Raymundo H, Scher AM, O'Leary DS, et al. Cardiovascular control by arterial and cardiopulmonary baroreceptors in awake dogs with atrioventricular block. Am. J. Physiol. Heart Circ. Physiol 1989; vol. 257:H2048-H2058.

4. Mukkamala R, Kim JK, Li Y, et al. Estimation of arterial and cardiopulmonary total peripheral resistance baroreflex gain values: validation by chronic arterial baroreceptor denervation. Am. J. Physiol. Heart Circ. Physiol 2006;vol. 290:H1830-H1836. [PubMed: 16306216]

5. Bainbridge FA. The influence of venous filling upon the rate of the heart. J Physiol 1915; vol. 50(no. 2):65-84. [PubMed: 16993330]

6. Sagawa K. The end-systolic pressure-volume relation of the ventricle: definition, modifications and clinical use. Circulation 1981;vol. 63:1223-1227. [PubMed: 7014027] 
7. Spratt JA, Tyson GS, Glower DD, et al. The end-systolic pressure-volume relationship in conscious dogs. Circulation 1987; vol. 75:1295-1309. [PubMed: 3568332]

8. Suga H, Sagawa K, Shoukas AA. Load independence of the instantaneous pressure-volume ratio of the canine left ventricle and effects of epinephrine and heart rate on the ratio. Circ. Res 1973; vol. 32:314-322. [PubMed: 4691336]

9. Cheng CP, Igarashi Y, Little WC. Mechanism of augmented rate of left ventricular filling during exercise. Circ. Res 1992;vol. 70:9-19. [PubMed: 1345778]

10. Chen X, Sala-Mercado JA, Hammond RL, et al. Dynamic control of maximal ventricular elastance in conscious dogs before and after pacing-induced heart failure.

11. Berger RD, Akselrod S, Gordon D, et al. An efficient algorithm for spectral analysis of heart rate variability. IEEE Trans Biomed Eng 1986; vol. 33(no. 9):900-904. [PubMed: 3759126]

12. Ljung, L. System Identification: Theory for the User. Prentice Hall: Englewood Cliffs; 1987.

13. Kawada T, Sato T, Inagaki M, et al. Closed-loop identification of carotid sinus baroreflex transfer characteristics using electrical stimulation. Jpn J Physiol 2000;vol. 50(no. 3):371-380. [PubMed: 11016987]

14. Kubota T, Alexander J Jr, Itaya R, et al. Dynamic effects of carotid sinus baroreflex on ventriculoarterial coupling studied in anesthetized dogs. Circ. Res 1992;vol. 70:1044-1053. [PubMed: 1568296] 

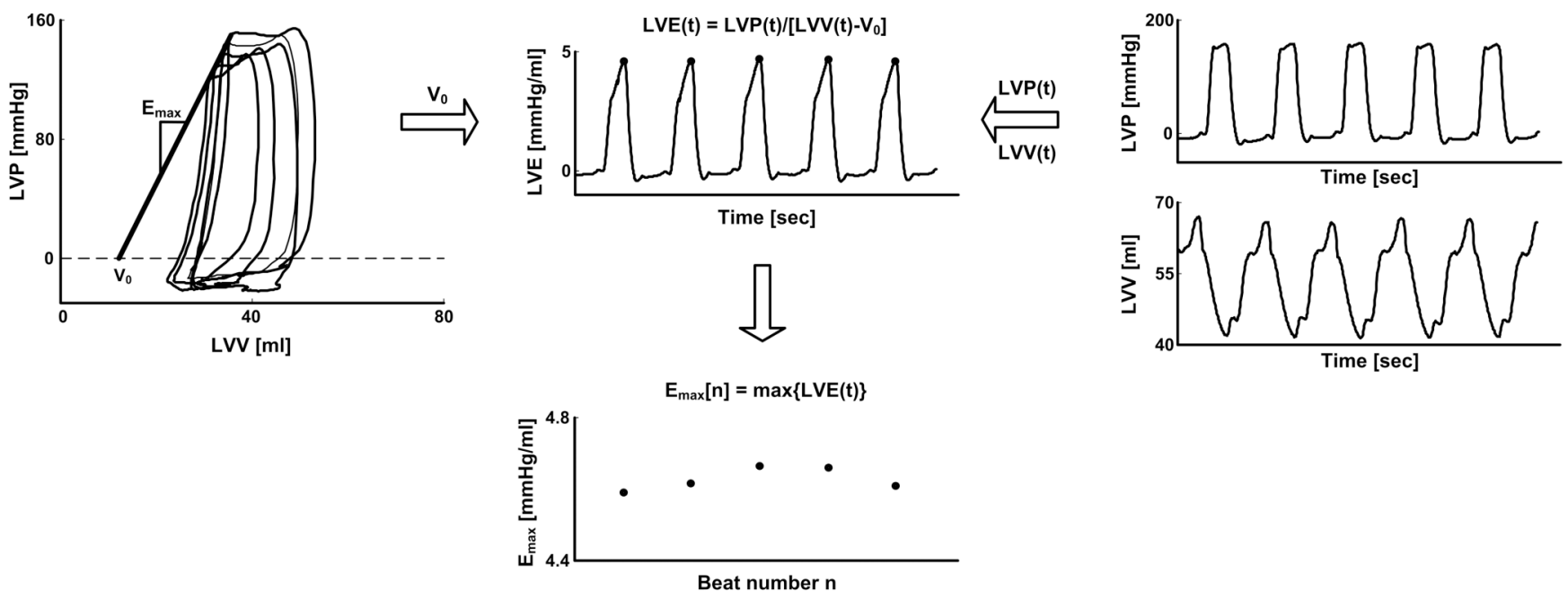

Fig. 1.

Procedure for estimating beat-to-beat $\mathrm{E}_{\max }$ from continuous LVP and LVV measurements during transient vena cava occlusion (left panel) and a baseline period (right panel). 


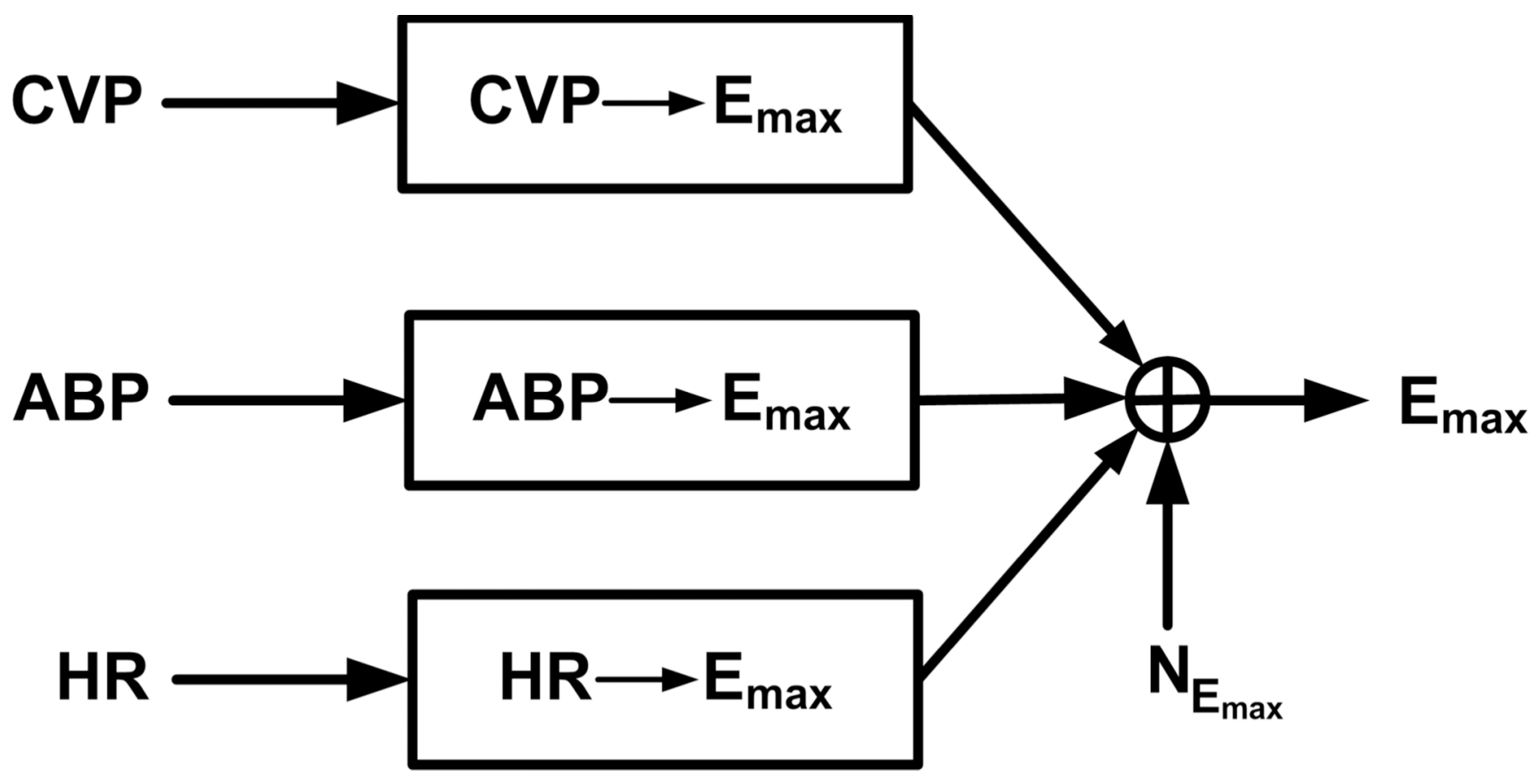

Fig. 2.

Block diagram for identifying the $\mathrm{CVP} \rightarrow \mathrm{E}_{\max }, \mathrm{ABP} \rightarrow \mathrm{E}_{\max }$ and $\mathrm{HR} \rightarrow \mathrm{E}_{\max }$ transfer functions from beat-to-beat fluctuations in CVP, ABP, HR, and $\mathrm{E}_{\max }$. 


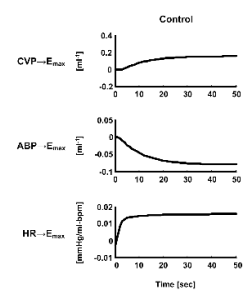

Fig. 3.

Transfer functions in terms of step responses from the first dog during the control condition. 


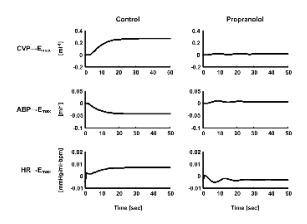

Fig. 4.

Transfer functions in terms of step responses from the second dog during the control and propranolol conditions. 